KN13 Extending the reach of molecular replacement. Randy J Read, Airlie J McCoy, Gábor Bunkóczi, Robert D Oeffner, Dept. of Haematology, Cambridge Institute for Medical Research, University of Cambridge, Cambridge, U.K.

E-mail: rir27@cam.ac.uk

We are developing our computer program Phaser [1] to implement maximum likelihood methods for solving macromolecular crystal structures by either molecular replacement (MR) or single-wavelength anomalous diffraction (SAD) phasing. The use of maximum likelihood is important for two reasons. First, likelihood targets are more sensitive in solving difficult MR problems than traditional Patterson-based methods. Second, likelihood scores for different possible solutions can be compared directly, providing a convenient basis for automated decision making. By combining MR and SAD in a single program, we have been able to develop convenient algorithms that use one source of phase information to bootstrap another [2]. Recent developments are aimed at extending the use of molecular replacement to more difficult problems, e.g. using poorer models or smaller fragments, and simplifying the application of methods combining molecular replacement with experimental phasing. For example, by combining the likelihood-based methods in Phaser with advanced modelling techniques in Rosetta, it has been possible both to solve structures with poorer starting templates and to extend the convergence radius of subsequent model-building and refinement [3]. The signal in difficult MR searches can be improved by using an ensemble of distantly-related templates, and MR with density cut out from one crystal form can be used to solve a second crystal form, thus initiating non-crystallographic symmetry averaging [4].

[1] McCoy, A.J., Grosse-Kunstleve, R.W., Adams, P.D., Winn, M.D., Storoni, L.C. \& Read, R.J. (2007). J. Appl. Cryst. 40, 658-674.

[2] Read, R.J. \& McCoy, A.J. (2011). Acta Cryst. D67: 338-344.

[3] DiMaio, F., Terwilliger, T.C., Read, R.J., Wlodawer, A., Oberdorfer, G., Wagner, U., Valkov, E., Alon, A., Fass, D., Axelrod, H.L., Das, D., Vorobiev, S.M., Iwad', H., Pokkuluri, P.R. \& Baker, D. (2011). Nature 473, 540-543.

[4] Zhou, A., Carrell, R.W., Murphy, M.P., Wei, Z., Yan, Y., Stanley, P.L.D., Stein, P.E., Broughton Pipkin, F. \& Read, R.J. (2010). Nature 468, 108-111.

Keywords: maximum likelihood; molecular replacement; single-wavelength anomalous diffraction

\section{KN14 Charge Densities and Crystal Engineering}

Mark A. Spackman, School of Chemistry \& Biochemistry, University of Western Australia, Australia

E-mail: mark.spackman@uwa.edu.au

The development of molecular crystalline materials with desired chemical or physical properties underpins many technological advances. Successful design of these materials demands knowledge and understanding at three different levels:

- the structure and properties of the constituent molecules;

- how these properties lead to observed crystal structures; and

- the relationship between molecular properties and those of the bulk material.

Many different aspects can be identified with each of these levels of understanding. For example, computational chemistry and experimental charge density analysis can provide information on molecular properties. Crystal structure prediction typically uses theoretical models for molecular charge distributions (in the form of atomic charges and multipoles, or as a discrete pixellated breakdown of the electron distribution) to evaluate the electrostatic components of interaction enrgies.

Our research in recent years has touched on aspects of all three of these levels, especially the relationship between molecular properties, crystal structure and bulk properties. The common thread in all of our studies has been a detailed appreciation of the nature of the molecular electron distribution. We are particularly interested in how and where it differs from that due to a simple sum of spherical atoms (the promolecule), and devising ways in which the two can be used together to facilitate understanding of how molecules pack in crystals, why it makes sense that a particular crystal packing occurs, and what can be learned about the bulk in this manner

This presentation will provide an overview of our latest research involving Hirshfeld surface analysis, [1] electrostatic complementarity [2] and the mapping of void space in molecular crystals.[3]

[1] Spackman, M. A. \& Jayatilaka, D. (2009). CrystEngComm 11, 19-32.

[2] Spackman, M. A., McKinnon, J. J. \& Jayatilaka, D. (2008). CrystEngComm 10, 377-388.

[3] Turner, M. J., McKinnon, J. J., Jayatilaka, D. \& Spackman, M. A. (2011). CrystEngComm 13, 1804-1813.

Keywords: charge density studies; crystal engineering; Hirshfeld surface analysis 